\title{
TbRGG2 facilitates kinetoplastid RNA editing initiation and progression past intrinsic pause sites
}

\author{
MICHELLE L. AMMERMAN, VLADIMIR PRESNYAK, ${ }^{1}$ JOHN C. FISK, BARDEES M. FODA, and LAURIE K. READ \\ Department of Microbiology and Immunology, University at Buffalo School of Medicine, Buffalo, New York 14214, USA \\ Witebsky Center for Microbial Pathogenesis and Immunology, University at Buffalo School of Medicine, Buffalo, New York 14214, USA
}

\begin{abstract}
TbRGG2 is an essential kinetoplastid RNA editing accessory factor that acts specifically on pan-edited RNAs. To understand the mechanism of TbRGG2 action, we undertook an in-depth analysis of edited RNA populations in TbRGG2 knockdown cells and an in vitro examination of the biochemical activities of the protein. We demonstrate that TbRGG2 down-regulation more severely impacts editing at the $5^{\prime}$ ends of pan-edited RNAs than at their $3^{\prime}$ ends. The initiation of editing is reduced to some extent in TbRGG2 knockdown cells. In addition, TbRGG2 plays a post-initiation role as editing becomes stalled in TbRGG2depleted cells, resulting in an overall decrease in the $3^{\prime}$ to $5^{\prime}$ progression of editing. Detailed analyses of edited RNAs from wildtype and TbRGG2-depleted cells reveal that TbRGG2 facilitates progression of editing past intrinsic pause sites that often correspond to the $3^{\prime}$ ends of cognate guide RNAs (gRNAs). In addition, noncanonically edited junction regions are either absent or significantly shortened in TbRGG2-depleted cells, consistent with impaired gRNA transitions. Sequence analysis further suggests that TbRGG2 facilitates complete utilization of certain gRNAs. In vitro RNA annealing and in vivo RNA unwinding assays demonstrate that TbRGG2 can modulate RNA-RNA interactions. Collectively, these data are consistent with a model in which TbRGG2 facilitates initiation and 3' to 5' progression of editing through its ability to affect gRNA utilization, both during the transition between specific gRNAs and during usage of certain gRNAs.
\end{abstract}

Keywords: RNA editing; trypanosome; RNA binding; mitochondria; RG domain

\section{INTRODUCTION}

Trypanosomes undergo a unique process of post-transcriptional RNA editing in which uridines are inserted into or deleted from mitochondrial pre-mRNAs to form translatable mRNAs. Mitochondrial RNAs can be separated into three groups: never edited RNAs, minimally edited RNAs that are only edited in a small region, and pan-edited RNAs that are edited essentially throughout the entire RNA. The sequence information for editing is transferred from mitochondrially encoded guide RNAs (gRNAs) that are complementary to the mature mRNA, through base-pairing interactions. Most gRNAs are encoded on minicircle DNA and act in trans on maxicircle-encoded mRNAs; however, the COII mRNA contains its gRNA in its $3^{\prime} \mathrm{UTR}$ and therefore the gRNA

\footnotetext{
${ }^{1}$ Present address: Department of Biochemistry, Case Western Reserve University School of Medicine, Cleveland, Ohio 44106, USA.

Reprint requests to: Laurie K. Read, Department of Microbiology and Immunology and Witebsky Center for Microbial Pathogenesis and Immunology, University at Buffalo School of Medicine, Buffalo, NY 14214, USA; e-mail: lread@buffalo.edu; fax: (716) 829-2158.

Article published online ahead of print. Article and publication date are at http://www.rnajournal.org/cgi/doi/10.1261/rna.2285510.
}

functions in cis. gRNAs are composed of three regions. The 5' anchor region hybridizes to the $3^{\prime}$ end of the complementary pre-mRNA. The $3^{\prime}$ end of the gRNA is composed of a posttranscriptionally added oligo $(\mathrm{U})$ tail that helps stabilize the gRNA-pre-mRNA interaction (Blum and Simpson 1990). The central region of the gRNA is the guiding sequence that directs the insertion and deletion of uridines into the mRNA. On pan-edited RNAs, multiple gRNAs act sequentially from $3^{\prime}$ to $5^{\prime}$ across large editing domains, and the newly edited sequence is complementary to the anchor region of the proximal gRNA (Maslov and Simpson 1992). Editing is catalyzed by the $20 \mathrm{~S}$ editosomes, multiprotein complexes that contain all the proteins necessary for cleavage of the premRNA, the addition or deletion of uridines, and the ligation of the newly edited RNA (for reviews, see Simpson et al. 2004; Lukes et al. 2005; Stuart et al. 2005). Recent work suggests that there may be three classes of 20 S editosomes, with both shared and unique subunits, each with distinct roles in the editing process (Panigrahi et al. 2006; Carnes et al. 2008; Ernst et al. 2009).

Additional noneditosome proteins are required for efficient editing because of the complex protein-RNA and 
RNA-RNA interactions involved in the editing process. MRP1/2 (Tb11.55.0009 and Tb11.01.4860) and RBP16 (Tb11.02.5770) are editing accessory factors that are specifically required for editing of the minimally edited apocytochrome b (CYb) transcript (Pelletier and Read 2003; Vondruskova et al. 2005; Fisk et al. 2009). MRP1/2 and RBP16 also stabilize a number of transcripts (Pelletier and Read 2003; Vondruskova et al. 2005; Fisk et al. 2009). Results of biochemical studies suggest MRP1/2 and RBP16 affect editing prior to the enzymatic steps of the reaction, potentially by facilitating gRNA/pre-mRNA association (Muller et al. 2001; Muller and Goringer 2002; Aphasizhev et al. 2003; Miller et al. 2006; Schumacher et al. 2006; Ammerman et al. 2008; Zikova et al. 2008).

Recently, a multiprotein complex, called the mitochondrial RNA binding complex 1 (MRB1 complex, also known as the gRNA-binding complex, or GRBC), was identified and shown to contain proteins essential for gRNA stability, mRNA stability, and RNA editing, as well as many proteins with an as-of-yet-undefined function (Hashimi et al. 2008; Weng et al. 2008; Acestor et al. 2009). Tandem affinity purification and mass spectrometry studies revealed that the MRB1 complex contains at least 14 components (Hashimi et al. 2008; Panigrahi et al. 2008; Weng et al. 2008; Hernandez et al. 2009). Protein-protein interactions in the MRB1 complex appear to be both RNA-dependent and RNA-independent, although further characterization of this complex is required (Weng et al. 2008; Acestor et al. 2009). Two proteins present in the complex, called GRBC1 and 2 (or GAP2 (Tb927.7.2570) and GAP1 (Tb927.2.3800)), are essential for growth in both the procyclic form (PF) and bloodstream form (BF) of the Trypanosoma brucei life cycle stages. GRBC1 and GRBC2 bind to gRNAs and are essential for the stability of minicircle-encoded gRNAs (Weng et al. 2008; Hashimi et al. 2009). Consequently, the depletion of GRBC1 and GRBC2 results in decreased levels of all edited transcripts except COII. Another component of the MRB1 complex, REH2 (Tb927.4.1500), that contains a $\mathrm{DExD} / \mathrm{H}$ helicase domain is also essential for growth in the PF and is required for gRNA stability (Hashimi et al. 2009; Hernandez et al. 2009). However, the entire MRB1 complex does not function in gRNA stability, since depletion of Tb11.01.7290, which contains a NUDIX hydrolase domain, affects edited mRNA stability in the absence of any change in gRNA levels (Weng et al. 2008; Hashimi et al. 2009). This phenotype is similar to that of another component of some MRB1 complexes, TbRGG1 (Tb927.6.2230), which may also affect the stability of edited mRNAs (Hashimi et al. 2008; Weng et al. 2008). Other components of the MRB1 complex, Tb927.6.1680 (C2H2 zinc finger) and Tb11.02.5390 (no known motif), are required for the growth of PF cells and affect the abundance of various mitochondrial mRNAs, likely at the level of editing; however, no specific function has been attributed to these proteins (Acestor et al. 2009).
Another protein that is present in MRB1 complex purifications is TbRGG2 (Tb10.406.0050) (Hashimi et al. 2008; Panigrahi et al. 2008; Weng et al. 2008; Hernandez et al. 2009). TbRGG2 (previously TbRGGm) is an RNA binding protein that appears to be transiently associated with editosomes. The protein was initially identified by mass spectrometry in preparations of immunoaffinity-purified editosomes, although not with biochemically purified editosomes (Panigrahi et al. 2003), and a small fraction of the protein coimmunoprecipitates with editosomes (Fisk et al. 2008). TbRGG2 is associated with 40S RNA-dependent and 20S RNA-independent complexes as well as smaller complexes (Fisk et al. 2008). It appears to be important for MRB1 complex stability as a proportion of the complex, as monitored by Western blotting for GRBC1, shifts to a lower $S$ value in glycerol gradients of TbRGG2-depleted cells (Acestor et al. 2009). TbRGG2 is essential for growth in both the PF and BF of T. brucei (Fisk et al. 2008). Down-regulation of TbRGG2 by RNAi results in massive editing defects in both life cycle stages. Interestingly, these defects, while profound, are restricted to pan-edited RNAs (Fisk et al. 2008; Acestor et al. 2009). Since the hallmark of pan-edited RNAs is the necessity for coordinated sequential usage of a large number of gRNAs, we hypothesize that TbRGG2 might affect this process.

Here, we utilize in vivo and in vitro methods to determine the mechanism of TbRGG2 activity during RNA editing and to understand the specific effect of TbRGG2 on pan-edited RNAs. We show that the effect of TbRGG2 depletion on the editing of pan-edited RNAs is much less severe at the $3^{\prime}$ ends of RNAs than at their $5^{\prime}$ ends. Both pre-edited and partially edited RNAs accumulate in TbRGG2 RNAi cells, as shown by poisoned primer extension (PPE) and PCR assays, indicating an effect of TbRGG2 on both editing initiation and progression. Detailed sequence analysis of pan-edited RNAs in TbRGG2 RNAi cells confirms that $3^{\prime}$ to $5^{\prime}$ progression of editing along pan-edited RNAs is significantly impacted upon TbRGG2 down-regulation, and reveals that the disruption in editing progression caused by TbRGG2 depletion is due primarily to blockage of editing at intrinsic pause sites that are evident in wild-type cells but exacerbated in TbRGG2 RNAi cells. Pause sites often correspond to the $3^{\prime}$ ends of gRNAs but can also be found within region directed by a single gRNA. In addition, noncanonically edited junction regions are significantly shorter or absent in TbRGG2-depleted cells. These data, together with the demonstration that gRNA levels are unaffected in TbRGG2depleted cells, are consistent with a role for TbRGG2 in gRNA utilization. In vitro RNA annealing and in vivo RNA unwinding assays demonstrate that TbRGG2 can modulate RNA-RNA interactions. Overall, these results support a model in which TbRGG2 is involved in the initiation of editing and progression of editing through intrinsic pause sites through modulation of gRNA/pre-mRNA interactions. 


\section{RESULTS}

\section{TbRGG2 does not affect total gRNA abundance}

TbRGG2 has been identified in pulldowns of the MRB1 multiprotein complex containing GRBC1 and 2 (also known as GAP2 and 1) (Hashimi et al. 2008; Weng et al. 2008). GRBC1 and 2 are gRNA binding proteins that are required for the stability of gRNAs, and depletion of either protein results in reduced total gRNA abundance (Weng et al. 2008; Hashimi et al. 2009). Additionally, TbRGG2 depletion specifically affects pan-edited transcripts, and their production relies on the utilization of multiple gRNAs. These factors led us to explore whether TbRGG2 is involved in gRNA biogenesis and/or stability. We obtained RNA from PF TbRGG2 RNAi cells that were uninduced or whose TbRGG2 expression was down-regulated by tetracycline induction for $3 \mathrm{~d}$ (Fisk et al. 2008). In these cells, the TbRGG2 protein levels were decreased $\sim 50 \%$ by day 2 post-induction and $77 \%$ by day 4 post-induction (Fisk et al. 2008). We confirmed a $>50 \%$ reduction in TbRGG2 protein levels on day 3 from all tetracycline-induced cells used in this study (data not shown). To determine if the depletion of TbRGG2 affects gRNA abundance, the total RNA from uninduced and induced TbRGG2 RNAi cells was incubated with $\alpha-\left[{ }^{32} \mathrm{P}\right] \mathrm{GTP}$ and vaccinia virus guanylyltransferase, which labels primary transcripts, including gRNAs (Blum and Simpson 1990). When the abundance of gRNAs was standardized to a T. brucei cytoplasmic RNA control, we observed no significant decrease in the gRNA levels upon TbRGG2 depletion (Fig. 1). While we cannot rule out that TbRGG2 may affect the stability of specific gRNAs, the absence of any perturbation of total gRNA levels demonstrates that

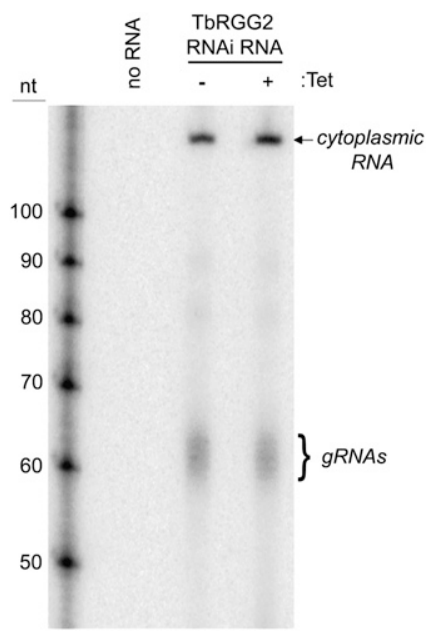

FIGURE 1. Depletion of TbRGG2 does not affect total gRNA levels. Guanylyltransferase labeling of gRNAs from the PF TbRGG2 RNAi cells at $72 \mathrm{~h}$ with or without tetracycline induction. Twenty micrograms of total RNA was labeled with $\left[\alpha-{ }^{32} \mathrm{P}\right]$ GTP using guanylyltransferase and resolved on a denaturing gel. RNA input was standardized against the quantity of labeled cytoplasmic RNA.
TbRGG2 plays a role in the MRB1 complex distinct from that of GRBC1 and 2.

\section{TbRGG2 depletion differentially affects RNA editing at the $3^{\prime}$ and $5^{\prime}$ ends of pan-edited RNAs}

We previously showed that the abundance of pan-edited RNAs is dramatically reduced in TbRGG2-depleted cells (Fisk et al. 2008). In those studies, we quantified edited RNAs by quantitative RT-PCR using primers specific to fully edited sequence at the $5^{\prime}$ end of each RNA, a method that detects RNAs that have completed or nearly completed the editing process (as diagramed in Figs. 2A, 3A). However, the production of a fully edited transcript can presumably be affected at numerous stages in the editing reaction, including both initiation and the $3^{\prime}$ to $5^{\prime}$ progression of editing. To begin to understand the function of TbRGG2 in the editing process, we analyzed the effect of TbRGG2 depletion on editing initiation using a PPE assay (Fig. 2A; Missel et al. 1997). If TbRGG2 is required for initiation of editing, facilitating processes such as association of the editosome with gRNA or pre-mRNA or catalysis of editing, we would expect to observe the same significant decrease in the levels of pan-edited RNAs using PPE as was observed with qRT-PCR. To address this issue, we first utilized a primer that anneals to the 3 ' never-edited region of RPS12, and included ddTTP in the reaction to halt extension following the first editing site. However, we were unable to extend this primer under these conditions (data not shown). We then created a primer that incorporates the first editing site (a $\mathrm{U}$ insertion) and a few extra nucleotides, allowing us to monitor editing at the second RPS12 editing site using ddTTP to halt extension. For simplification, we will still refer to this reaction as RPS12 initiation PPE, bearing in mind that initiation is much more likely to be limiting than is the transition from the first to the second editing site. We analyzed the pan-edited RPS12 RNA from TbRGG2 RNAi cells uninduced or induced for $3 \mathrm{~d}$ using PPE, normalizing the edited RNA signal to that of a tubulin PPE performed in the same reaction. As shown in Figure 2B (and Supplemental Fig. 1A), the abundance of edited RPS12 is reduced in the tetracycline-induced cells compared with the uninduced cells. Quantification of the edited RPS12 signals from duplicate initiation PPE (+ddT) experiments indicated that, upon TbRGG2 depletion, edited RPS12 RNA was reduced an average of $41 \pm 6 \%$ (Fig. 2D). These PPE results can be compared to our previously published qRTPCR results in which depletion of TbRGG2 for $3 \mathrm{~d}$ in PF $T$. brucei cells resulted in an $85 \pm 5 \%$ reduction in the levels of RPS12 edited RNA when normalized to tubulin RNA levels (Fig. 2D; Fisk et al. 2008). The difference in the extent of the editing defect caused by TbRGG2 down-regulation detected using PPE versus qRT-PCR assays presumably reflects the regions of the RNAs being analyzed for editing. As diagramed for the RPS12 RNA in Figure 2A, the qRT-PCR primers used to detect the edited transcripts anneal to the $5^{\prime}$ end of the 
A

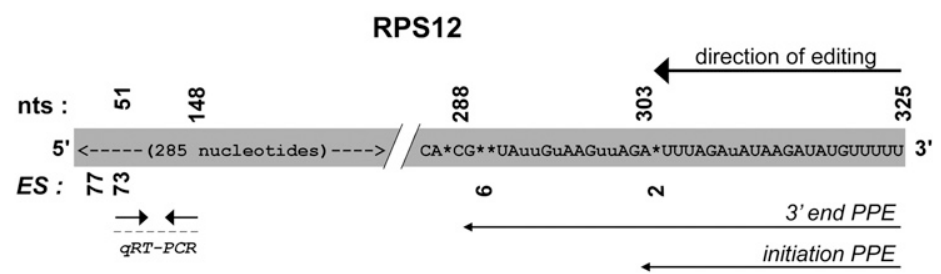

B

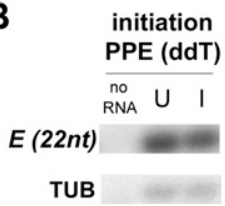

D

\begin{tabular}{rr}
\multicolumn{2}{c}{ Edited decrease } \\
\hline 5'end : & $85 \pm 5 \%$ \\
3'end : & $42 \pm 3 \%$ \\
\hline
\end{tabular}

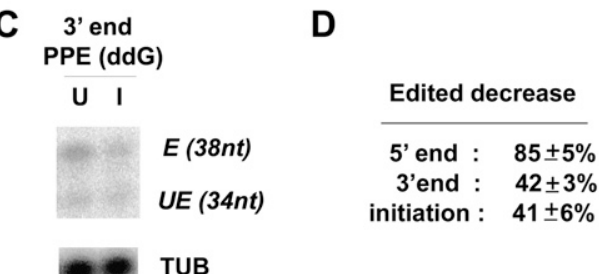

FIGURE 2. TbRGG2 differentially affects editing of pan-edited RPS12 RNA at the $3^{\prime}$ and $5^{\prime}$ ends. (A) Diagram of fully edited RPS12 RNA depicting the nucleotide position (top) and the number of edited sites (ESs; bottom) on the fully edited RNA. The regions of the RNA where editing is examined using qRT-PCR and poisoned primer extensions (PPEs) are labeled. $(B)$ Representative initiation PPE. The reaction was performed using radiolabeled primers that anneal to the $3^{\prime}$ end of RPS12 RNA from PF TbRGG2 RNAi cells after $72 \mathrm{~h}$ with (I) or without $(\mathrm{U})$ tetracycline induction. The products arising from extension of the edited (E) RNAs are labeled. Tubulin RNA levels (TUB) were analyzed by PPE in the same reaction as a control. (C) Representative $3^{\prime}$ end PPE. The reaction was performed using radiolabeled primers that anneal to the $3^{\prime}$ end of RPS12 RNA from PF TbRGG2 RNAi cells after $72 \mathrm{~h}$ with (I) or without (U) tetracycline induction. The products arising from extension of unedited (UE) and edited (E) RNAs are labeled. Tubulin RNA levels (TUB) were analyzed by PPE in the same reaction as a control. $(D)$ Comparison of the decrease in edited RNA levels following TbRGG2 loss using qRT-PCR to analyze $5^{\prime}$ fully edited transcripts (Fisk et al. 2008) and PPEs to analyze editing at initiation and near the $3^{\prime}$ ends of the transcripts. For the PPE reactions, the decreases in edited RNA levels were determined using RNA isolated from at least duplicate experiments. Edited RNA levels were normalized to tubulin PPEs performed in the same reaction.

strates a differential effect on editing at the $3^{\prime}$ and $5^{\prime}$ ends of the RPS12 pan-edited RNAs. The increased severity of the editing defect observed by qRT-PCR compared with those measured with either RPS12 PPE assay suggests that TbRGG2 is also affecting editing at a step subsequent to utilization of the first gRNA.

To determine if this differential effect of TbRGG2 depletion on the $5^{\prime}$ and $3^{\prime}$ ends of edited RPS12 also applies to other pan-edited RNAs, we analyzed the effect of TbRGG2 on editing initiation and at the 3 ' end of A6 RNA. As before, PPE was performed to analyze editing initiation. A primer specific to the $3^{\prime}$ never edited region of A6 was extended until the first adenine at nt 783 of 820 in the fully edited sequence; this adenine is just $5^{\prime}$ of the first ES (Fig. 3A). The quantification of the signals from duplicate initiation PPE $(+\mathrm{ddT})$ experiments normalized to an internal tubulin control indicated that, upon TbRGG2 depletion, A6 RNA was reduced an average of $29 \pm 16 \%$, indicating a modest effect on editing initiation similar to that observed with RPS12 RNA (Fig. 3B,D; Supplemental Fig. 2A). To analyze editing slightly further $5^{\prime}$, we performed the previously published A6

gene and amplify the sequence from nucleotides (nt) 51-148 of the fully edited RNA. Thus, amplification of "edited RPS12" by qRT-PCR would require complete editing at all but the four $5^{\prime}$ most edited sites (ESs; positions between nonuridine nucleotides that are edited in the final transcript), or 73 of 77 total ESs. In contrast, the initiation PPE $(+\mathrm{ddT})$ analyzes the edited RNA from the $3^{\prime}$ end to the first adenine $5^{\prime}$ of the second ES at nt 303 of 325 in the fully edited RNA (or nt 199 of 221 in the unedited RNA), as diagramed in Figure 2A. To further quantify editing near the $3^{\prime}$ end of RPS12 RNA, we performed a PPE reaction that analyzes the edited RNA from the $3^{\prime}$ end to the first cytosine (using ddGTP), which is $5^{\prime}$ of the sixth ES at nt 288 of the fully edited RNA (see Fig. 2A). Similar to the initiation PPE, there was a $42 \pm 3 \%$ reduction in the levels of RPS12 edited RNA, suggesting that the editing decrease observed by $3^{\prime}$ end PPE (+ddG) terminating $5^{\prime}$ of ES 6 was caused by events at or prior to editing at ES 2 (Fig. 2D). Both of the PPE reactions monitor editing of the RPS12 transcript within the region whose editing is specified by the first gRNA, as predicted by the KISS (kinetoplastid RNA editing sequence search tool) database (Ochsenreiter et al. 2007); thus, utilization of this first RPS12 gRNA appears to be impaired $\sim 40 \%$ upon TbRGG2 depletion. In addition, comparison of the PPE and qRT-PCR results for TbRGG2 RNAi cells also demon- $3^{\prime}$ end PPE (+ddG) (Pelletier and Read 2003), which analyzes the edited RNA from the $3^{\prime}$ end to the first cytosine $5^{\prime}$ of the primer at nt 688 of 820 in the fully edited RNA (or nt 320 of 401 in the unedited RNA). This region, which contains the initial 25 ESs out of a total of 185 ESs (Fig. 3A), would likely be encoded by three sequential gRNAs based on the length of the region and known gRNA binding sites (Bhat et al. 1990; Koslowsky et al. 1992; Shu et al. 1995). Quantification of the signals from triplicate PPE experiments indicated that, upon TbRGG2 depletion, edited A6 RNA was reduced an average of $48 \pm 5 \%$ (Fig. 3C,D; Supplemental Fig. 2B). These PPE results stand in contrast to the $92 \pm 4 \%$ reduction in the levels of A6 edited RNA by qRT-PCR (Fig. 3D; Fisk et al. 2008). As diagramed for the A6 RNA in Figure 3A, the qRTPCR primers used to detect the edited transcripts anneal to the $5^{\prime}$ end of the gene and amplify the sequence from nt 55198 of the fully edited RNA. Thus, amplification of "edited A6" by qRT-PCR would require complete editing at all but the five $5^{\prime}$ most ESs, or 180 of 185 total ESs. Therefore, analyses of A6 editing by PPE and qRT-PCR demonstrate a progressive decrease in editing as levels are monitored from the $3^{\prime}$ to the $5^{\prime}$ end (Fig. 3D). Overall, comparison of PPE and qRT-PCR results for TbRGG2 RNAi cells demonstrates a differential effect on editing at the $3^{\prime}$ and $5^{\prime}$ ends of the A6 and RPS12 pan-edited RNAs. 
A

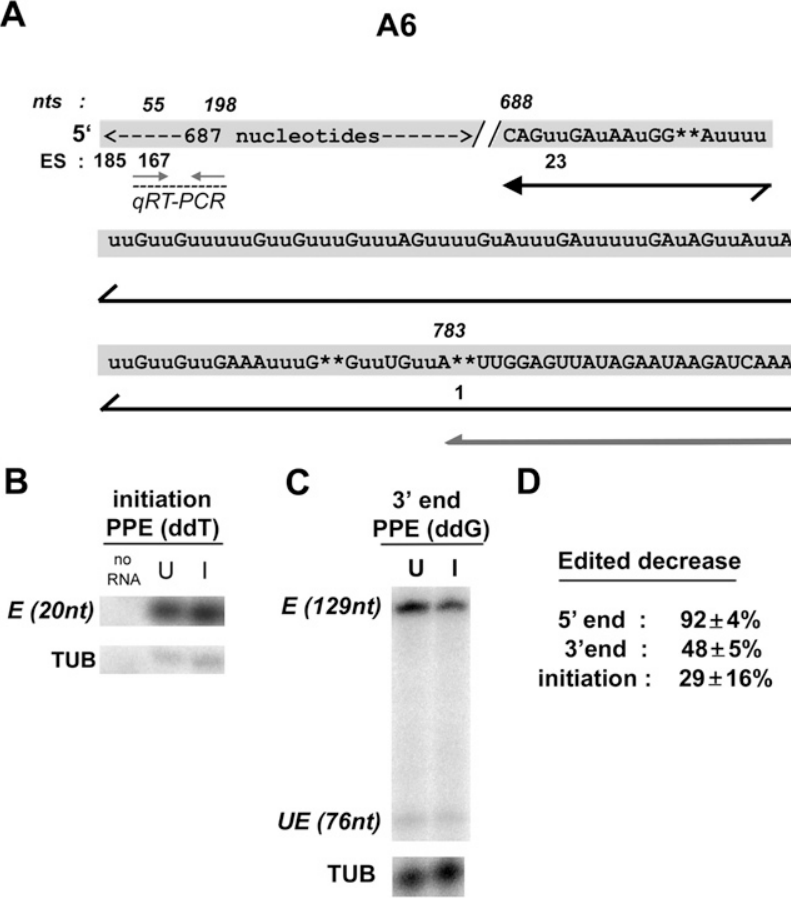

FIGURE 3. TbRGG2 differentially affects editing of pan-edited A6 RNA at the $3^{\prime}$ and $5^{\prime}$ ends. (A) Diagram of fully edited A6 RNA depicting the nucleotide position (top) and the number of edited sites (ESs; bottom) on the fully edited RNA. The regions of the RNA where editing is examined using qRT-PCR and poisoned primer extensions (PPEs) are labeled. $(B, C)$ Representative initiation PPEs $(B)$ and $3^{\prime}$ end PPEs $(C)$ were performed as described in Figure 2 but with primers specific to A6. $(D)$ Comparison of the decrease in edited RNA levels following TbRGG2 loss using qRT-PCR to analyze $5^{\prime}$ fully edited transcripts (Fisk et al. 2008) and PPEs to analyze editing at initiation and near the $3^{\prime}$ ends of the transcripts. For the PPE reactions, the decreases in edited RNA levels were determined using RNA isolated from at least duplicate experiments. Edited RNA levels were normalized to tubulin PPEs performed in the same reaction.

\section{TbRGG2 affects $3^{\prime}$ to $5^{\prime}$ progression of editing}

Having seen a quantitative difference in the effect of TbRGG2 depletion on RNA editing at the $3^{\prime}$ and $5^{\prime}$ ends of pan-edited transcripts, we next asked whether TbRGG2 affects the $3^{\prime}$ to $5^{\prime}$ progression of editing. The steady-state populations of RNAs that undergo pan-editing consist of varying amounts of unedited, partially edited, and fully edited RNAs, which can be distinguished by size. To analyze the complete populations of A6 and RPS12 RNAs, we performed RTPCR analysis using primers that anneal to the small neveredited regions at the $5^{\prime}$ and $3^{\prime}$ ends of the A6 and RPS12 RNAs, which flank the large edited regions. Because of experimental limitations, the $3^{\prime}$ primer for RPS12 RT-PCR also contains the first ES. This method allows us to compare the relative amounts of unedited, partially edited, and fully edited transcripts on the same gel (Schnaufer et al. 2001; Ochsenreiter et al. 2008b). When RT-PCR reactions performed with RNA from uninduced cells are compared to those with RNAs from tetracycline-induced cells, we observe a clear difference in the relative levels of the different classes of RNAs. In both A6 and RPS12 RT-PCR reactions, there is a significant decrease in the amount of fully edited RNAs when TbRGG2 is depleted, as expected (Fig. 4). More importantly, we observe a relative increase in the proportion of partially edited RNAs within each population upon TbRGG2 depletion. The sizes of the RNAs that accumulate in TbRGG2-depleted cells suggest that the editing of pan-edited RNAs is stalled, resulting in an accumulation of RNAs with less editing than in uninduced cells. For A6, a major product is observed at $\sim 490 \mathrm{bp}$. This product appears to reflect RNA that is edited to just past the region monitored by 3 ' end PPE (Fig. 3A), based on the size of the PCR product, which is slightly larger than would be produced by the 50 Us inserted in the edited RNA monitored by PPE (although size cannot be a direct indication of the extent of editing due to inclusion of both $U$ insertions and deletions and the presence of junction regions) (see below and Koslowsky et al. 1991; Sturm and Simpson 1991; Sturm et al. 1992). These data are consistent with a role for TbRGG2 in the $3^{\prime}$ to $5^{\prime}$ progression of editing.

We next cloned and sequenced the RPS12 RT-PCR reactions products uninduced versus induced TbRGG2 RNAi cells, reasoning that the specific nature of editing pause sites in TbRGG2depleted cells could illuminate the protein's function. Because there is a bias for small insert clones, we chose RPS12 for analysis since the unedited transcript is only $221 \mathrm{nt}$ and the fully edited RNA is $325 \mathrm{nt}$. Fifty-one clones were obtained using the RPS12 RT-PCR products from the uninduced cells, and 46 of these sequences were edited to some degree. Cloning of RT-PCR products obtained from TbRGG2-depleted cells resulted in 64 clones, 58 of which were edited to some degree. Sequences from clones containing some degree of editing were aligned to the fully edited RPS12 sequence, and the fully edited region of each clone is schematically diagramed in Figure 5. In both uninduced and induced cells, there appear to be common sites at which full editing stalls. Nevertheless, comparisons of the relative degree of full editing in cDNAs from uninduced and induced cells confirms that, within the population, editing progresses a further $5^{\prime}$ on RNAs obtained from cells with wild-type TbRGG2 levels. For example, a significant number of cDNAs were obtained from both populations in which the fully edited region ends at or $3^{\prime}$ of nt 285 (Fig. 5, right-hand arrows), and this population was increased in induced relative to uninduced cells. We observed $45 \%$ of cDNAs ( 26 of 58 ) with a $3^{\prime}$ proximal fully edited region ending at or $3^{\prime}$ of nt 285 in the induced cells compared to $33 \%$ (15 of 46) 

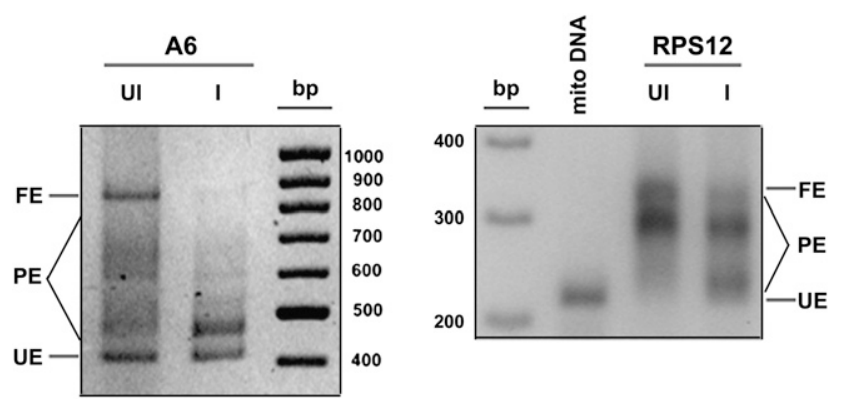

FIGURE 4. Depletion of TbRGG2 results in an increased pool of partially edited RNAs. Gel analysis of RT-PCR reactions using RNAs from TbRGG2 RNAi cells that were induced (I) and uninduced (UI) for $72 \mathrm{~h}$. Primers to the RPS12 and A6 genes are specific to nucleotides outside of the edited sequence and amplify the entire population including unedited (UE), partially edited (PE), and fully edited (FE) RNAs. Mitochondrial (Mito) DNA was used as a template control for sizing the unedited RPS12 transcript.

of cDNAs in uninduced cells. These data are consistent with increased stalling at an early stage of RPS12 RNA editing in the absence of TbRGG2. RPS12 appears to contain a second major fully edited endpoint that occurs around nt 150 (Fig. 5, left-hand arrows). We found that a smaller percentage of RPS12 cDNAs contained fully edited sequence $5^{\prime}$ of this point in TbRGG2-depleted cells (7\%) compared with uninduced cells (26\%). There also appear to be more pausing in the induced cells between RPS12 nt 150 and 285. These results demonstrate that depletion of TbRGG2 results in a decreased $3^{\prime}$ to $5^{\prime}$ progression of editing and increased termination of fully edited regions at specific sites.

The sequences diagramed in Figure 5 represent only the extent of the fully edited sequence in each clone. However, partially edited RNAs typically contain junction regions between the 3' fully edited and the 5' unedited regions. Junctions are composed of edited sequence that differs from the mature edited sequence, referred to here and previously (Guo et al. 2010) as noncanonical edited sequence. Junctions vary greatly in length and can substantially increase the total size of the RNA. It is presumably due to differences in junction lengths that the full editing stall points so apparent in Figure 5 are not reflected in the gel analysis of the total cDNA population shown in Figure 4. Junction regions are proposed to result from the guiding of editing by a noncognate gRNA or through the interaction of the cognate gRNA with an inappropriate upstream region of the RNA (Koslowsky et al. 1991; Sturm and Simpson 1991; Sturm et al. 1992). While RNAs with junction regions could be dead-end products, given the prevalence of such RNAs, junctions most likely represent regions of RNAs that are undergoing editing at the time of isolation. In Figure 6A, we again show a diagram of the RPS12 cDNA clones; however, in Figure 6A, the sequences are arranged by total length, rather than by the length of fully edited sequence, as in Figure 5, and both the junction regions (light gray) and the fully edited regions (dark gray) are shown. In addition to differences in the extent of fully edited sequence shown in Figure 5, we observed a dramatic difference in the lengths of junction regions between RPS12 cDNAs from uninduced and induced TbRGG2 RNAi cells (Fig. 6A). These differences are quantified in Figure 6B, where we defined the length of the junction as the number of potential editing sites (PESs) within the junction sequence. Here, PES refers to positions between all nonuridine nucleotides. The junction begins at the site where the sequence switches from fully edited to preedited or noncanonical edited sequence, and continues until the transcript contains continuous pre-edited sequence. The percentage of RNAs that lack a junction region in the uninduced cells was $13.2 \%$, and this was increased in the TbRGG2-depleted cells to 29.3\%. Moreover, the proportion of RNAs with smaller junctions of 10 PESs or less is significantly increased in induced versus uninduced cells ( $41.3 \%$ vs. $22.6 \%$ ), while the proportion with longer junctions of $>10$ PESs is correspondingly decreased upon TbRGG2 depletion (29.3\% in induced vs. $64.2 \%$ in uninduced). Thus, ablation of TbRGG2 results in production of RNAs with shorter or nonexistent junction regions.

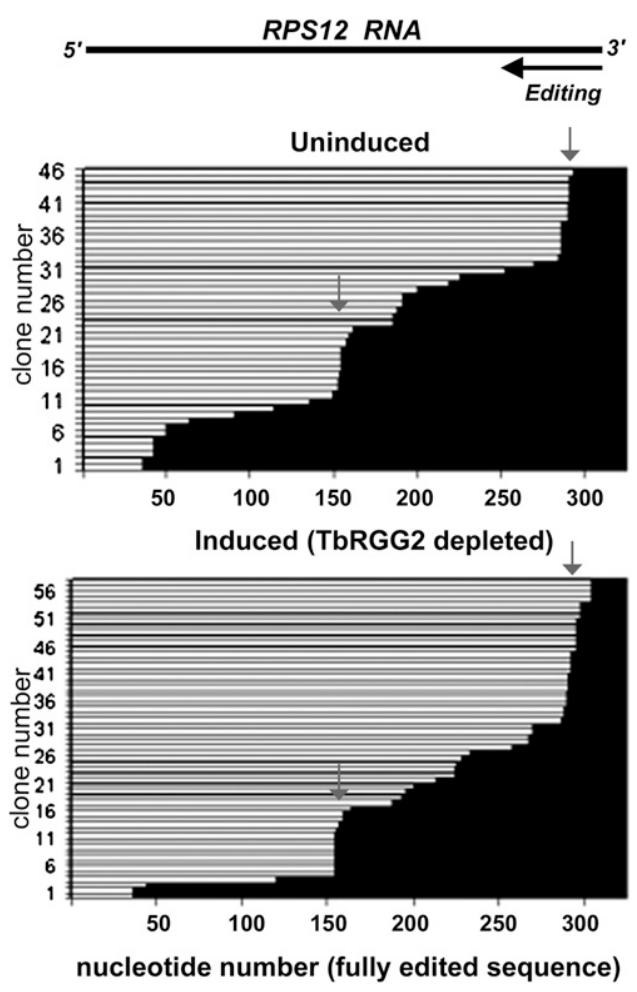

FIGURE 5. Analysis of cDNA sequences obtained from RT-PCR amplification of RPS12 RNAs from TbRGG2 RNAi cells. RPS12 RTPCR reactions shown in Figure 4 were cloned and sequenced. Black bars represent regions of fully edited sequence in RPS12 cDNAs relative to the fully edited RPS12 sequence. The nucleotide number of the fully edited sequence is shown on the bottom. cDNAs isolated from uninduced cells are shown in the top diagram, and those from induced cells are shown in the bottom diagram. Completely unedited sequences were omitted from the graph. Gray arrows indicate the predominant stall sites around nucleotides 154 and 285 . 


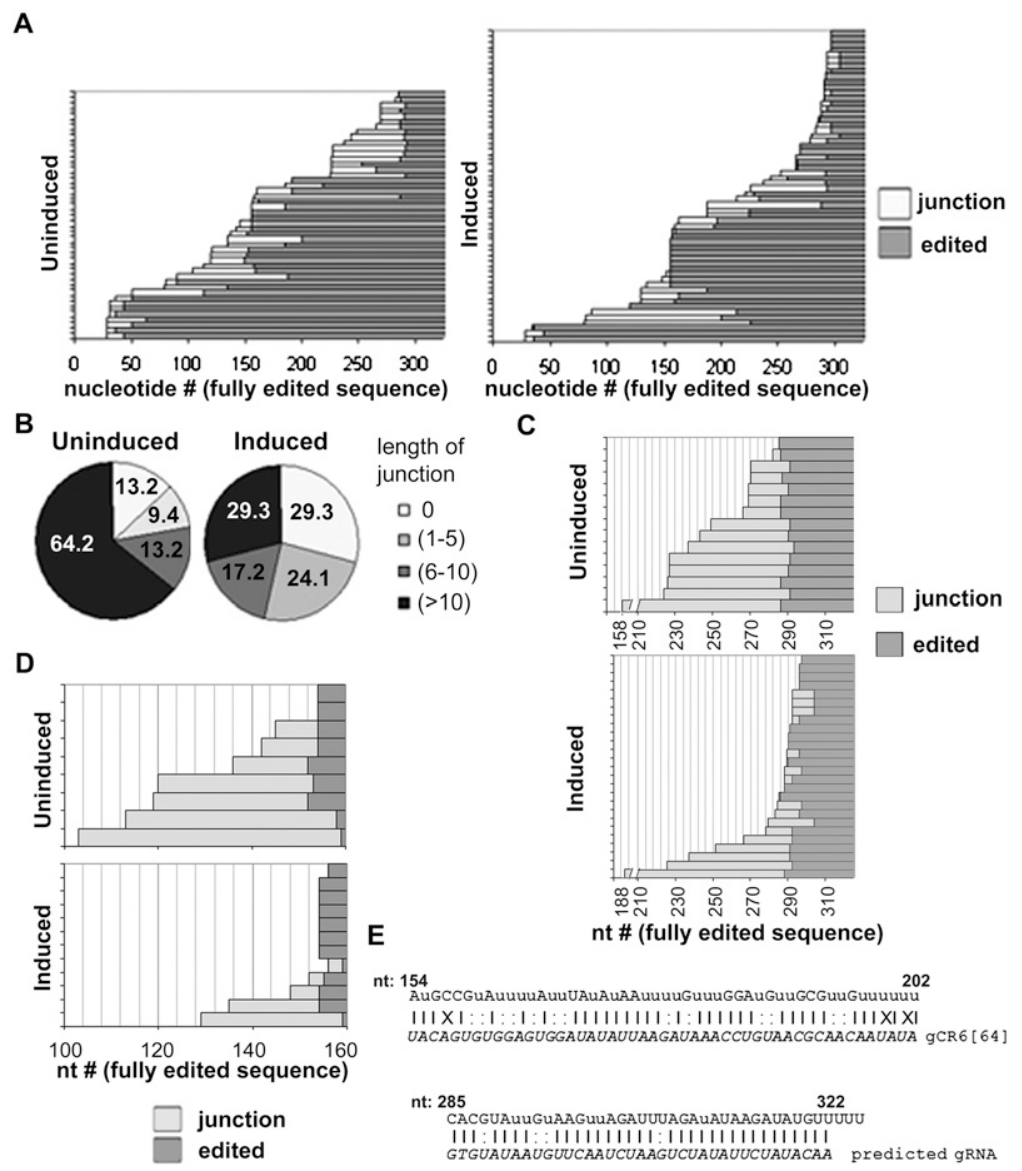

FIGURE 6. Depletion of TbRGG2 results in shorter junction regions in RPS12 RNAs. $(A)$ Graphic representation of RPS12 cDNAs cloned and sequenced from RT-PCR reactions shown in Figure 4. Dark gray represents fully edited sequence, and light gray represents junction region. The nucleotide number of the fully edited sequence is shown on the bottom. cDNAs isolated from uninduced cells are shown in the left diagram, and those from induced cells are shown in the right diagram. Completely unedited sequences were omitted from the graph. $(B)$ Quantitation of the length of junction regions in RPS12 clones shown in A. The length of the junction is defined as the number of potential editing sites (PESs), positions between nonuridine nucleotides, within the sequence. The percentage of clones represented by each slice is shown in the pie graph. $(C)$ Graphic representation of a portion of the sequences of all RPS12 cDNA clones in which full editing terminates before nucleotide (nt) 285 in uninduced (top) and induced (bottom) TbRGG2 RNAi cells. Edited indicates fully edited sequence. $(D)$ Graphic representation of a portion of the sequences of all RPS12 cDNA clones in which full editing terminates between nt 150 and 160 in uninduced (top) and induced (bottom) TbRGG2 RNAi cells. (E) Regions of the edited RPS12 mRNA, where editing preferentially accumulates, aligned with cognate gRNA. The gRNA, gCR6[64], is aligned on the bottom of the RPS12 mRNA region with editing terminating at nt 154 (top). A predicted gRNA is aligned on the bottom to the RPS12 mRNA region with editing terminating at nt 285 (bottom). In the RPS12 sequence, uppercase letters represent bases present in the DNA and lowercase u's represent uridines added during editing. ( $\mid$ ) represents a Watson-Crick base pair; (:) represents a G-U base pair; and $\mathrm{X}$ represents a mismatch.

Closer inspection of Figure 6A suggested that most of the differences in junction lengths occurred within the two major pause sites around nt 150 and nt 285. For instance, of the 15 cDNAs from uninduced cells that terminate full editing at or $3^{\prime}$ of nt 285 , all but two contain junction regions that extend much further $5^{\prime}$ past nt 285 (Fig. 6C, top). In contrast, 17 of the 26 cDNAs from induced cells whose full editing terminates at or $3^{\prime}$ of nt 285 have either no junction or junction regions that terminate $3^{\prime}$ of nt 285 (Fig. $6 \mathrm{C}$, bottom). Similar results were observed in clones in which full editing terminates between nt 150 and 160 , with an increased number of clones displaying no or shorter junctions in cells in TbRGG2-depleted cells (Fig. 6D). Interestingly, we found that in TbRGG2depleted cells, we obtained multiple clones ( $s i x$ of 58 edited RNAs) from three independent RT-PCR reactions, containing identical sequence. In these cDNAs, editing terminates precisely at nt 154 and there is no junction region (Fig. 6D, bottom). Two clones with this sequence were also obtained from the uninduced cells (Fig. 6D, top). However, overall we found that cDNAs from uninduced cells with fully edited sequence ending around nt 154 displayed significantly more heterogeneity in their editing end points and the sizes of the subsequent junction regions than did cDNAs from induced cells.

To address a potential role of gRNA/ mRNA interactions in the generation of pause sites, we more closely examined the RPS12 editing pause sites from the data set displayed in Figures 5 and 6 in the context of predicted cognate gRNAs. Notably, nt 154 aligns precisely with the $3^{\prime}$ end of a predicted gRNA, gCR6[64] (Fig. 6E, top; Read et al. 1992). That is, a significant number of RPS12 clones that accumulate in TbRGG2-depleted cells are fully edited through the region specified by gCR6[64], and they lack a subsequent junction region, suggesting that editing is halted precisely after the editing directed by this gRNA. The termination of the fully edited region in multiple clones at the end of a gRNAencoded region and the absence of a junction region in these clones suggest that TbRGG2 is involved in modulating this gRNA/mRNA interaction and the transition to utilization of the proximal gRNA. The other major region of fully edited sequence termination that was preferentially observed in TbRGG2-depleted cells was at or before nt 285. This is in the vicinity of the $3^{\prime}$ end of a potential gRNA (at nt 285) predicted from minicircle sequences in the KISS database (Fig. 6E, bottom; Ochsenreiter et al. 2007). Importantly, Figure 6C shows that induced TbRGG2 RNAi cells contain an increased proportion of clones whose fully edited sequence terminates prior to the site specified by the $3^{\prime}$ end of this 
potential gRNA. This suggests that complete utilization of this gRNA may be compromised in TbRGG2-depleted cells. Collectively, these data support a role for TbRGG2 in gRNA utilization, during both the transition between specific gRNAs and during usage of certain gRNAs.

\section{TbRGG2 modulates RNA-RNA interactions}

The effect of TbRGG2 on both initiation of editing and the $3^{\prime}$ to $5^{\prime}$ progression of editing may result from its influence on the regulation or utilization of gRNAs and/or mRNAs. Notably, we observed no significant decrease in the steadystate levels of total gRNA upon TbRGG2 depletion (Fig. 1), suggesting an effect on RNA utilization. We previously reported that TbRGG2 binds both gRNAs and mRNAs in vitro (Fisk et al. 2008). Here, we wanted to characterize the additional biochemical activities of TbRGG2 related to RNA to provide insight into its role in RNA editing. The editing accessory factor RBP16, like TbRGG2, contains an RGG domain, and it has previously been shown that this domain possesses RNA annealing activity and can act on a gRNA/ pre-mRNA pair (Ammerman et al. 2008). To determine if TbRGG2 can stimulate formation of a gRNA/pre-mRNA hybrid, we performed in vitro annealing assays with a truncated pre-edited A6 mRNA molecule (41-nt A6U5) and its cognate gRNA, gA6[14]NX, as previously described (Ammerman et al. 2008). Little annealing was seen in the absence of protein (Fig. 7, lane 2), or in the presence of 2.5 $\mu \mathrm{M}$ of the negative control protein, $\mathrm{p} 22$, a mitochondrial protein that does not bind RNA (lane 9) (Hayman et al. 2001). RBP16 was used as a positive control, and micromolar amounts stimulated hybrid formation as previously shown (Fig. 7, lane 8). In the same assay, addition of increasing amounts of TbRGG2 stimulated gRNA/pre-mRNA annealing up to 15-fold at nanomolar concentrations (Fig. 7). Interestingly, higher concentrations of TbRGG2 became inhibitory to RNA annealing. Stimulation of RNA annealing by TbRGG2 is specific to cognate RNA pairs as the protein does not facilitate annealing of $\mathrm{gCYb}[558]$, a noncognate gRNA, to 41-nt A6U5 (Fig. 7, lane 10). These results demonstrate that TbRGG2 possesses RNA annealing activity and can stimulate formation of a gRNA/pre-mRNA hybrid.

In addition to facilitating RNA annealing, many proteins modulate RNA structure by unwinding RNA. In fact, stimulation of RNA annealing can result from the ability of a protein to unwind RNA secondary structure, thereby facilitating biamolecular hybrid formation. To determine if TbRGG2 can melt RNA secondary structure, we performed an in vivo assay that tests for transcription anti-termination and is an indirect assay for RNA unwinding (Phadtare et al. 2002; Rajkowitsch et al. 2005; Kim et al. 2007). We utilized the Esherichia coli strain RL211, which contains a trpL terminator that forms a hairpin structure upstream of a cat gene (Fig. 8A). If the RL211 cells express a protein with RNA unwinding activity, these cells are able to express chloram-
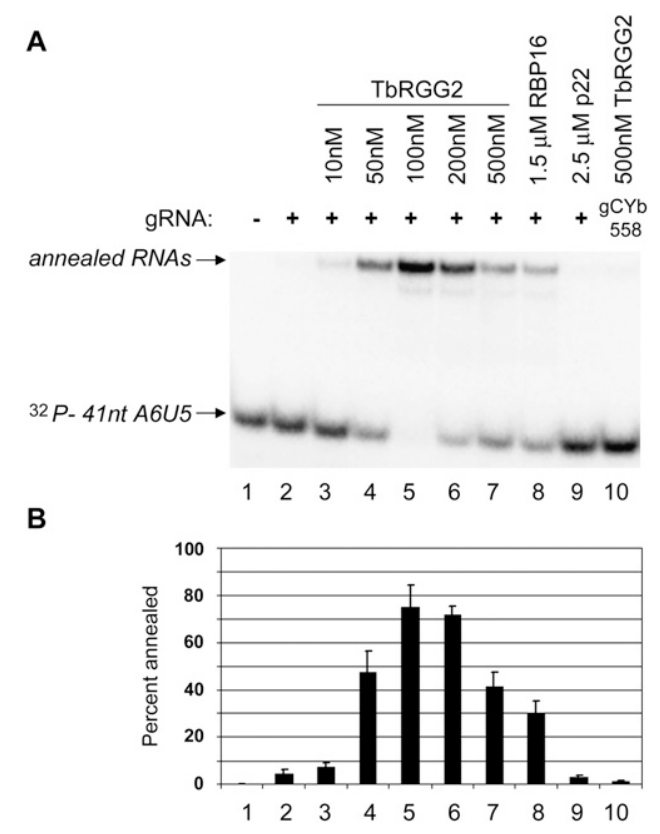

FIGURE 7. Recombinant TbRGG2 stimulates annealing of A6 premRNA to cognate gRNA in vitro. $(A)$ Representative annealing assay with radiolabeled A6U5 41nt mRNA and gA6[14]NX gRNA at the indicated protein concentrations. (Lane 1) A6U5 pre-mRNA alone, (lane 2) A6U5 mRNA and gA6[14]NX gRNA in the absence of protein, (lanes 3-7) cognate gRNA/mRNA pair in the presence of increasing TbRGG2, (lane 8 ) RBP16 positive control, (lane 9) p22 negative control, (lane 10) reaction with TbRGG2 and a noncognate gRNA/mRNA pair. $(B)$ The bar graph represents the mean and SE of the percentage of the input pre-mRNA that is annealed to the gRNA in at least three separate experiments.

phenicol acetyltransferase, allowing for growth in the presence of chloramphenicol (Bae et al. 2000). As shown in Figure $8 \mathrm{~B}$, cells containing the empty PINIII vector can grow on LBampicillin in the absence of chloramphenicol but are unable to grow on LB-ampicillin with chloramphenicol. As a positive control, we show that cells overexpressing bacterial CspE are able to grow in the presence of chloramphenicol, as previously shown (Phadtare et al. 2002). When RL211 cells expressing TbRGG2 were tested in this assay, they were also able to grow in the presence of chloramphenicol. These data demonstrate that TbRGG2 possesses transcription anti-termination activity resulting from its ability to melt an RNA duplex. Thus, TbRGG2 can modulate RNA-RNA interactions by facilitating both RNA annealing and unwinding.

\section{DISCUSSION}

Previous studies in our laboratory identified TbRGG2 as an RNA editing accessory factor that is essential for growth in both the PF and BF life cycle stages (Fisk et al. 2008). Quantitative RT-PCR showed that depletion of TbRGG2 results in a specific reduction in the levels of pan-edited RNAs, while levels of never edited, minimally edited, and 
A

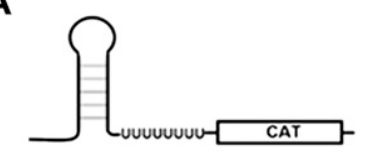

B

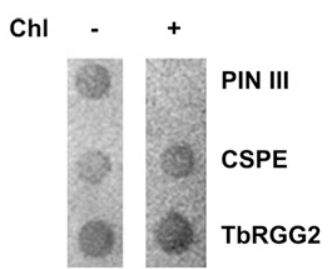

FIGURE 8. TbRGG2 can unwind RNA. (A) E. coli strain RL211 contains the trpL terminator that forms a hairpin loop upstream of a chloramphenicol (chl) resistance reporter gene (CAT). (B) RL211 cells expressing empty pINIII vector, CspE (positive control), or TbRGG2 were grown in LB with $100 \mu \mathrm{g} / \mathrm{mL}$ ampicillin and $1 \mathrm{mM}$ IPTG for $1 \mathrm{~h}$ to an approximate $\mathrm{OD}_{600}$ of 1 and spotted on LB plates containing $50 \mu \mathrm{g} / \mathrm{mL}$ ampicillin and $1 \mathrm{mM}$ IPTG, with and without $15 \mu \mathrm{g} / \mathrm{mL}$ chl. Cells were grown at $37^{\circ} \mathrm{C}$ for $1 \mathrm{~d}(-\mathrm{chl})$ or $4 \mathrm{~d}(+\mathrm{chl})$.

pre-edited versions of pan-edited RNAs are increased. To provide insight into the mechanism of TbRGG2 action and to understand why TbRGG2 specifically affects editing of pan-edited RNAs, we undertook an in-depth analysis of edited RNA populations in TbRGG2-depleted cells. Several lines of evidence presented here indicate that TbRGG2 facilitates initiation of editing as well as the $3^{\prime}$ to $5^{\prime}$ progression of editing past intrinsic editing pause sites on an mRNA. Our data are consistent with a model in which TbRGG2 affects gRNA utilization, during the transition between specific gRNAs and during usage of certain gRNAs.

An effect on editing processivity could be manifested by one of the following mechanisms: enhancing progression of the editosome from one editing site to the next, facilitating usage of individual and/or sequential gRNAs, or coordinating access of the RNAs to different editosome subtypes (e.g., transfer between insertion and deletion sites). Comparison of partially edited RPS 12 cDNA sequences from uninduced and induced TbRGG2 RNAi cells showed that depletion of TbRGG2 results in the editing pausing with higher frequency at specific sites. Since a lesser degree of pausing was observed at similar sites in uninduced cells, these are sites at which editing pauses intrinsically during the editing of this RNA. We also observed a decrease in the length and frequency of the subsequent junction regions. Comparison of RPS12 partially edited mRNA sequences with cognate gRNA sequences revealed that sites where editing preferentially terminated correspond to sites specified by the $3^{\prime}$ ends of two predicted gRNAs. These results suggest a role for TbRGG 2 in the transition between gRNAs on a pre-mRNA. The depletion of TbRGG2 does not affect the transition between all gRNAs equally, however, suggesting that there are specific gRNAs whose removal and/or recruitment is particularly sensitive to TbRGG2 levels. It is unlikely that gRNAs or mRNAs interact with TbRGG2 in a sequencespecific manner, based on the previously defined RNA binding characteristics of the protein (Fisk et al. 2008). Rather, gRNAs that require TbRGG2 for their efficient usage may represent low abundance gRNAs, gRNAs that act subsequent to especially stable gRNA/pre-mRNA pairs, or gRNAs destined to interact with regions of mRNA secondary structure. In addition to its role in facilitating the transition between gRNAs, TbRGG2 also appears to facilitate editing progression within the region of transcript encoded by a single gRNA. Again, not all gRNA specified regions are equally affected; pause sites likely reflect regions of inefficient gRNA/pre-mRNA annealing and a resultant inability of that gRNA to direct editing of the cognate mRNA.

Biochemical analyses of TbRGG2 are also consistent with a role in facilitating gRNA/mRNA interaction. We show that TbRGG2 possesses the ability to both anneal RNAs and unwind RNA secondary structure. These activities could contribute to gRNA utilization at multiple steps that are not mutually exclusive. For example, TbRGG2 might directly participate in unwinding the gRNA/mRNA duplex, such that the anchor sequence of the proximal gRNA can be formed. It may also directly facilitate the formation of the gRNA/mRNA anchor duplex through its RNA annealing activity. Finally, TbRGG2 could indirectly contribute to gRNA/mRNA interactions by resolving intramolecular structure in gRNA and/or mRNA. Future studies will be needed to clarify these possibilities.

Other non-MRB1 editing accessory factors, MRP1/2 and RBP16, have also been shown to possess the ability to modulate RNA-RNA interactions, yet these proteins affect completely different mitochondrial transcripts and steps in the editing process than TbRGG2 (Muller et al. 2001; Muller and Goringer 2002; Aphasizhev et al. 2003; Pelletier and Read 2003; Vondruskova et al. 2005; Miller et al. 2006; Schumacher et al. 2006; Ammerman et al. 2008; Fisk et al. 2008, 2009; Zikova et al. 2008). These differences presumably reflect the fact RNA editing is a complex process, requiring a large number of specific $\mathrm{RNA}$-protein and RNA-RNA interactions. Because of this complexity, it is likely numerous proteins that modify inter- and intramolecular RNA structure are involved in the editing process at multiple steps.

Recently, Guo et al. (2010) reported that mutation of the zinc finger domain of the core editosome subunit KREPA3, like TbRGG2, affected the progression of editing. In cells harboring KREPA3 with a zinc finger mutation, PCR analysis revealed a decrease in fully edited A6 RNA and an increase in corresponding partially edited RNA. Interestingly, RT-PCR analysis revealed a major partially edited A6 RNA product that accumulated near the $3^{\prime}$ end of the transcript, similar to the results we observe following depletion of TbRGG2 (Fig. 4). These investigators also noted that the partially edited A6 mRNAs that accumulate in the mutant cells are present at 
lower frequency in wild-type cells (Guo et al. 2010), suggesting these positions are intrinsic pause sites.

The occurrence of intrinsic pause sites in uninduced TbRGG2 cells suggests that progression past these sites is normally a rate-limiting step in editing, and explains why an editing accessory factor is required. Therefore, in addition to addressing TbRGG2 function, the results from this study also provide insight into the general mechanism of RNA editing. We note that even in uninduced cells, the utilization of all gRNAs does not result in equivalent pauses in editing. Again, this suggests that certain gRNAs or gRNA transitions have characteristics that necessitate the action of an accessory factor. Deep sequencing of RNA populations from wild-type cells will be needed to better understand the kinetic and thermodynamic restrictions that affect the various steps in editing on different transcripts. Similar analyses of knockdown cell lines will provide insight into the mechanisms by which these steps are influenced by core editosome proteins and editing accessory factors, including TbRGG2.

Junctions regions composed of long stretches of noncanonically edited sequence between unedited and fully edited regions of an mRNA are typically observed in steadystate RNA populations from kinetoplastid mitochondria. Junctions have been proposed to result from misguiding by an incorrect gRNA or misalignment of the correct gRNA (Koslowsky et al. 1991; Sturm et al. 1992). It has been suggested that the junction region results from progressive realignment of the gRNA with the mRNA being edited and that $3^{\prime}$ junction boundaries may be defined by the site of gRNA/pre-mRNA anchor duplex formation (Koslowsky et al. 1991). Repeated re-editing of junction regions as directed by cognate gRNA is thought to ultimately result in fully edited sequence. In TbRGG2-depleted cells, we observe a substantial overall decrease in junction lengths, which further supports a role for TbRGG2 in the progression past pause sites resulting from altered gRNA/mRNA interaction. If TbRGG2 is involved in RNA unwinding and removal of a gRNA from mRNA, its absence may result in an increased stability of gRNA/mRNA pairs. That is, in TbRGG2-depleted cells, gRNAs tend to stay associated with mRNAs until they have completed or nearly completed direction of full editing, and even then their release may be delayed. This scenario would be expected to result in partially edited mRNAs with shorter or absent junctions regions, and this is what we observed in TbRGG2-depleted cells.

Interestingly, the pause sites in uninduced cells at which full editing terminates are frequently sites where the sequence transitions to long junctions. Because the significance of these long junction regions is not well understood, the possibility remains that they do not arise simply from realignment of a single cognate gRNA or usage of an incorrect gRNA. Another possibility is that junction regions reflect utilization of multiple cognate gRNAs that direct the production of alternatively edited mRNAs. These alternatively edited RNAs are proposed to increase protein diversity
(Ochsenreiter and Hajduk 2006; Ochsenreiter et al. 2008a,b). Inherently inefficient gRNA transitions may cause the editing machinery to pause, thereby providing it an opportunity to sample a larger population of cognate gRNAs. Alternatively, pause sites may be defined by the presence of multiple cognate gRNAs, and the editosome may pause while determining which gRNA to utilize. In either of these models, editing accessory factors such as TbRGG2 could regulate the outcome or efficiency of alternative RNA editing.

TbRGG2 is reported to be associated with the MRB1 complex, which functions in mitochondrial RNA metabolism (Hashimi et al. 2008; Panigrahi et al. 2008; Acestor et al. 2009). Acestor et al. (2009) reported that depletion of TbRGG2 affects the MRB1 complex stability, as monitored by sedimentation of GRBC1 in glycerol gradients. However, it is unknown whether TbRGG2 is simply connected to GRBC2 by an RNA bridge or whether it serves as a central protein in a GRBC2-containing complex that becomes destabilized upon TbRGG2 depletion. Because TbRGG2 is a component of the MRB1 complex, we cannot rule out that some of the observed effects of TbRGG2 depletion may be indirect, such as an impact on helicase recruitment. However, functional analysis indicates TbRGG2 itself can modulate RNA-RNA interactions. TbRGG2 depletion does not affect gRNA stability; thus, it differs in its function from the GRBC1/2 proteins (Weng et al. 2008; Hashimi et al. 2009). Moreover, depletion of TbRGG2 leads to different effects on mitochondrial mRNAs compared with some other characterized MRB1 complex proteins (Weng et al. 2008; Acestor et al. 2009; Hashimi et al. 2009). Together, these findings suggest a complex level of structural and functional interactions in the MRB1 complex that are coordinated in part through various classes of RNAs. Additional characterization of the role of TbRGG2 in the MRB1 complex is required to understand the RNA-dependent and RNA-independent interactions between MRB1 proteins and how these relate to their functional interactions.

\section{MATERIALS AND METHODS}

\section{Guanylyltransferase assay}

Guanylyltransferase labeling was performed with total RNA from the TbRGG2 mutant RNAi cells uninduced or induced for $3 \mathrm{~d}$ with $2.5 \mu \mathrm{g} / \mathrm{mL}$ of tetracycline. RNA was purified from cells using Trizol, and the contaminating DNA was degraded using the DNAfree kit (Ambion) according to the manufacturer's instructions. Twenty micrograms of RNA was labeled with $5 \mu \mathrm{Ci}\left[\alpha-{ }^{32} \mathrm{P}\right]$ GTP $(800 \mathrm{Ci} / \mathrm{mmol})$ in the presence of $1.5 \mathrm{pmol}$ guanylyltransferase $\mathrm{DIR}^{1-545} 50 \mathrm{mM}$ Tris-HCl (pH 8), $1.5 \mathrm{mM} \mathrm{MgCl}_{2}, 6 \mathrm{mM} \mathrm{KCl}, 2.5$ $\mathrm{mM} \mathrm{DTT}$, and $20 \mathrm{U}$ SUPERasein for $30 \mathrm{~min}$ at room temperature (Hayman and Read 1999). Reactions were phenol chloroformextracted twice, chloroform-extracted once, and precipitated in the presence of $2 \mathrm{M}$ ammonium acetate, $20 \mu \mathrm{g}$ of glycogen, and ethanol. Samples were resuspended in $16 \mu \mathrm{L}$ of $90 \%$ formamide 
loading buffer, and half of each sample was resolved on an $8 \%$ acrylamide/7 $\mathrm{M}$ urea gel in $1 \times \mathrm{TBE}$.

\section{Poisoned primer extension}

The following radiolabeled primers (restriction sites are underlined) were used to analyze editing at the $3^{\prime}$ end:

A6 3'NE (5-GCGGATCCATTTGATCTTATTCTATAACTCC-3)

CR6-13-GP (5-AAAAACATATCTTAT-3)

TUB-RT-GP (5-GGGGGTCGCACTTTGTC-3).

The following radiolabeled primers were used to analyze editing initiation:

RPS12-RT-new (5-AAAACATATCTTATATCT-3)

TUB-RT-new \#2 (5-ACCCTCACCGACGTACCAGTGCACGA AGG-3)

A6-new-RT (5-TCTTATTCTATAACTCC-3).

For PPE, total RNA was purified from at least two biological replicates of uninduced and tetracycline $(2.5 \mu \mathrm{g} / \mathrm{mL})$ induced PF TbRGG2 RNAi cells at 72-h post-induction, as described previously (Fisk et al. 2008). PPE was performed with $15 \mu \mathrm{g}$ of RNA and 10 pmol $5^{\prime}{ }^{32} \mathrm{P}$-labeled primer. For $3^{\prime}$ end PPE reactions, primers were annealed to RNA samples by heating for $5 \mathrm{~min}$ at $95^{\circ} \mathrm{C}$, cooling on ice for $5 \mathrm{~min}$, and annealing for $60 \mathrm{~min}$ at $37^{\circ} \mathrm{C}$ in buffer with $10 \mathrm{mM}$ Tris (pH 8.0), $1 \mathrm{mM}$ EDTA, and $120 \mathrm{mM}$ $\mathrm{KCl}$. Extension reactions were performed in $50 \mathrm{mM}$ Tris- $\mathrm{HCl}(\mathrm{pH}$ 8.3); $50 \mathrm{mM} \mathrm{KCl} ; 10 \mathrm{mM} \mathrm{MgCl}_{2} ; 5.5 \mathrm{mM}$ DTT; $150 \mu \mathrm{M}$ each dATP, dCTP, and dTTP; $75 \mu \mathrm{M}$ ddGTP; $20 \mathrm{U}$ of RNasin (Promega); and $10 \mathrm{U}$ of AMV reverse transcriptase (Fermentas) for $45 \mathrm{~min}$ at $42^{\circ} \mathrm{C}$. RNA was digested with RNase A (2 $\left.\mu \mathrm{g}\right)$ for $15 \mathrm{~min}$ at $37^{\circ} \mathrm{C}$ in the presence of $1 \mu \mathrm{g}$ salmon sperm DNA. Extension products were resolved on denaturing polyacrylamide gels and analyzed by autoradiography followed by densitometry using Quanitiy One software (Bio-rad).

PPE reactions analyzing editing initiation were performed essentially as described above except ddTTP was used to terminate the extension. The primer RPS12-RT-new includes the first editing site and monitors editing at the second editing site; however, for simplification we will still refer to this as RPS12 initiation PPE. Tubulin control and edited RNA PPEs were performed simultaneously in the same reaction, and the levels of a given edited RNA were subsequently normalized to the tubulin RNA recovery control. The values for the decrease in edited product in the initiation and $3^{\prime}$ end PPEs were calculated as the average of the [(induced edited RNA value/induced tubulin value)/(uninduced edited RNA value/uninduced tubulin value) $] \times 100$ for each experiment. This method of calculation permits direct comparison of the effects of TbRGG2 depletion to those previously determined by qRT-PCR reactions where edited RNA levels were normalized to tubulin (Fisk et al. 2008).

\section{RT-PCR and sequence analysis of mitochondrial transcripts}

Thirty microliters of mid-log trypanosome culture were harvested and resuspended in $1 \mathrm{~mL}$ Trizol (Invitrogen). Total RNA was isolated according to the manufacturer's instructions. Contaminating DNA was removed from the RNA using the DNA-free kit (Ambion) and subsequent phenol-chloroform extraction. The primers used for RT-PCR anneal to the never edited $5^{\prime}$ and $3^{\prime}$ regions of the A6 gene and the $5^{\prime}$ never edited region of the RPS12 gene. The $3^{\prime}$ RPS12 Rev primer anneals to the $3^{\prime}$ end but includes the first ES (double underlined) to allow for efficient priming. The following primers were used for RT-PCR analysis (restriction site underlined):

A6 5' NE (5' GCGAATTCAAATAAGTATTTTGATATTATTAAAG 3') A6 3' (5' ATTAACTTATTTGATCTTATTCTATAACTCC 3)' RPS12 fwd (5' CTAATACACTTTTGATAACAAAC 3')

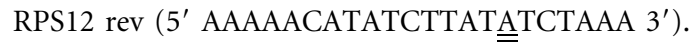

RT-PCR was performed essentially as previously described (Schnaufer et al. 2001). Briefly, 75 pmol of downstream primer for A6 or $100 \mathrm{pmol}$ oligo(dT) for RPS12 was annealed to $1 \mu \mathrm{g}$ of total RNA in a $10 \mu \mathrm{L}$ reaction by incubation for $5 \mathrm{~min}$ at $70^{\circ} \mathrm{C}$ and slow cooling $(30 \mathrm{~min})$ to $20^{\circ} \mathrm{C}$. The annealed primer was extended with Superscript III Reverse Transcriptase (Invitrogen) for $1 \mathrm{~h}$ at $42^{\circ} \mathrm{C}$. Seventy-five picomoles of upstream primer was added to the A6 PCR, and both upstream and downstream primers were added to the RPS12 PCR. PCR was performed using an annealing temperature of $45^{\circ} \mathrm{C}$. Mitochondrial DNA was used as a template control for sizing the unedited RPS12 transcript. PCR products were analyzed on a $2 \%-3 \%$ Agarose gels and cloned and sequenced by standard procedures (sequences are provided in Supplemental Figs. 3, 4).

\section{In vitro RNA annealing}

RNA annealing assays were performed as previously described (Ammerman et al. 2008) using the A6U5 41nt pre-mRNA and gA6[14]NX gRNA.

\section{In vivo RNA unwinding assay}

The RNA unwinding assay was performed essentially as described previously (Ammerman et al. 2008). TbRGG2 was amplified with primers TbRGG2 5'NdeI (GCGCATATGAAGCGCACACCTGTT AG) and TbRGGm 3-21 (GGAAGCTTTTACACCTTCTGACTGGC; restriction sites are underlined). TbRGG2 was digested with HindIII/NdeI and cloned into the pINIII vector cut at the same sites. To test TbRGG2 transcriptional anti-termination activity, the pINIII expression constructs were transformed into RL211 cells kindly provided by Dr. Robert Landick (University of Wisconsin-Madison) (Bae et al. 2000) and grown in LB with $100 \mu \mathrm{g} / \mathrm{mL}$ ampicillin overnight. The cells were then diluted into fresh media and grown to an $\mathrm{OD}_{600}$ of 0.4 , at which time IPTG was added to a final concentration of $1 \mathrm{mM}$, and cells were then grown for $1 \mathrm{~h}$. Cultures were spotted on LB plates containing 100 $\mu \mathrm{g} / \mathrm{mL}$ ampicillin and $1 \mathrm{mM}$ IPTG, with and without $15 \mu \mathrm{g} / \mathrm{mL}$ chloramphenicol, and were grown for $1-4 \mathrm{~d}$ at $37^{\circ} \mathrm{C}$.

\section{SUPPLEMENTAL MATERIAL}

Supplemental material can be found at http://www.rnajournal.org.

\section{ACKNOWLEDGMENTS}

We thank Dr. Sara Zimmer for critical reading of the manuscript. The kind gifts of yeast strain RL211 from Dr. Robert Landick and PINIII vector from Dr. Sangita Phadtare (Robert Wood Johnson 
Medical School) are greatly appreciated. This work was supported by National Institutes of Health grant RO1 AI061580 to L.K.R. M.L.A. was supported in part by Postdoctoral Fellowship 0725813T from the American Heart Association. B.M.F. was supported in part by a scholarship from the Egyptian Ministry of Higher Education.

Received May 26, 2010; accepted August 9, 2010.

\section{REFERENCES}

Acestor N, Panigrahi AK, Carnes J, Zikova A, Stuart KD. 2009. The MRB1 complex functions in kinetoplastid RNA processing. RNA 15: $277-286$.

Ammerman ML, Fisk JC, Read LK. 2008. gRNA/pre-mRNA annealing and RNA chaperone activities of RBP16. RNA 14: 1069-1080.

Aphasizhev R, Aphasizheva I, Nelson RE, Simpson L. 2003. A 100-kD complex of two RNA-binding proteins from mitochondria of Leishmania tarentolae catalyzes RNA annealing and interacts with several RNA editing components. RNA 9: 62-76.

Bae W, Xia B, Inouye M, Severinov K. 2000. Escherichia coli CspAfamily RNA chaperones are transcription antiterminators. Proc Natl Acad Sci 97: 7784-7789.

Bhat GJ, Koslowsky DJ, Feagin JE, Smiley BL, Stuart K. 1990. An extensively edited mitochondrial transcript in kinetoplastids encodes a protein homologous to ATPase subunit 6. Cell 61: 885-894.

Blum B, Simpson L. 1990. Guide RNAs in kinetoplastid mitochondria have a nonencoded $3^{\prime}$ oligo $(\mathrm{U})$ tail involved in recognition of the preedited region. Cell 62: 391-397.

Carnes J, Trotter JR, Peltan A, Fleck M, Stuart K. 2008. RNA editing in Trypanosoma brucei requires three different editosomes. Mol Cell Biol 28: 122-130.

Ernst NL, Panicucci B, Carnes J, Stuart K. 2009. Differential functions of two editosome exoUases in Trypanosoma brucei. RNA 15: 947957.

Fisk JC, Ammerman ML, Presnyak V, Read LK. 2008. TbRGG2, an essential RNA editing accessory factor in two Trypanosoma brucei life cycle stages. J Biol Chem 283: 23016-23025.

Fisk JC, Presnyak V, Ammerman ML, Read LK. 2009. Distinct and overlapping functions of MRP1/2 and RBP16 in mitochondrial RNA Metabolism. Mol Cell Biol 29: 5214-5225.

Guo X, Ernst NL, Carnes J, Stuart KD. 2010. The zinc-fingers of KREPA3 are essential for the complete editing of mitochondrial mRNAs in Trypanosoma brucei. PLoS ONE 5: e8913.

Hashimi H, Zikova A, Panigrahi AK, Stuart KD, Lukes J. 2008. TbRGG1, an essential protein involved in kinetoplastid RNA metabolism that is associated with a novel multiprotein complex. RNA 14: $970-980$.

Hashimi H, Cicova Z, Novotna L, Wen YZ, Lukes J. 2009. Kinetoplastid guide RNA biogenesis is dependent on subunits of the mitochondrial RNA binding complex 1 and mitochondrial RNA polymerase. RNA 15: 588-599.

Hayman ML, Read LK. 1999. Trypanosoma brucei RBP16 is a mitochondrial Y-box family protein with guide RNA binding activity. J Biol Chem 274: 12067-12074.

Hayman ML, Miller MM, Chandler DM, Goulah CC, Read LK. 2001. The trypanosome homolog of human p32 interacts with RBP16 and stimulates its gRNA binding activity. Nucleic Acids Res 29: 5216-5225.

Hernandez A, Madina BR, Ro K, Wohlschlegel JA, Willard B, Kinter MT, Cruz-Reyes J. 2009. REH2 RNA helicase in kinetoplastid mitochondria: Ribonucleoprotein complexes and essential motifs for unwinding and guide RNA binding. J Biol Chem 285: 12201228.

Kim JY, Park SJ, Jang B, Jung CH, Ahn SJ, Goh CH, Cho K, Han O, Kang H. 2007. Functional characterization of a glycine-rich RNA- binding protein 2 in Arabidopsis thaliana under abiotic stress conditions. Plant J 50: 439-451.

Koslowsky DJ, Bhat GJ, Read LK, Stuart K. 1991. Cycles of progressive realignment of gRNA with mRNA in RNA editing. Cell 67: 537546.

Koslowsky DJ, Riley GR, Feagin JE, Stuart K. 1992. Guide RNAs for transcripts with developmentally regulated RNA editing are present in both life cycle stages of Trypanosoma brucei. Mol Cell Biol 12: 2043-2049.

Lukes J, Hashimi H, Zikova A. 2005. Unexplained complexity of the mitochondrial genome and transcriptome in kinetoplastid flagellates. Curr Genet 48: 277-299.

Maslov DA, Simpson L. 1992. The polarity of editing within a multiple gRNA-mediated domain is due to formation of anchors for upstream gRNAs by downstream editing. Cell 70: 459-467.

Miller MM, Halbig K, Cruz-Reyes J, Read LK. 2006. RBP16 stimulates trypanosome RNA editing in vitro at an early step in the editing reaction. RNA 12: 1292-1303.

Missel A, Souza AE, Norskau G, Goringer HU. 1997. Disruption of a gene encoding a novel mitochondrial DEAD-box protein in Trypanosoma brucei affects edited mRNAs. Mol Cell Biol 17: 48954903.

Muller UF, Goringer HU. 2002. Mechanism of the gBP21-mediated RNA/RNA annealing reaction: matchmaking and charge reduction. Nucleic Acids Res 30: 447-455.

Muller UF, Lambert L, Goringer HU. 2001. Annealing of RNA editing substrates facilitated by guide RNA-binding protein gBP21. EMBO J 20: 1394-1404.

Ochsenreiter T, Hajduk SL. 2006. Alternative editing of cytochrome c oxidase III mRNA in trypanosome mitochondria generates protein diversity. EMBO Rep 7: 1128-1133.

Ochsenreiter T, Cipriano M, Hajduk SL. 2007. KISS: the kinetoplastid RNA editing sequence search tool. RNA 13: 1-4.

Ochsenreiter T, Anderson S, Wood ZA, Hajduk SL. 2008a. Alternative RNA editing produces a novel protein involved in mitochondrial DNA maintenance in trypanosomes. Mol Cell Biol 28: 5595-5604.

Ochsenreiter T, Cipriano M, Hajduk SL. 2008b. Alternative mRNA editing in trypanosomes is extensive and may contribute to mitochondrial protein diversity. PLoS ONE 3: e1566. doi: 10.1371/ journal.pone.0001566.

Panigrahi AK, Allen TE, Stuart K, Haynes PA, Gygi SP. 2003. Mass spectrometric analysis of the editosome and other multiprotein complexes in Trypanosoma brucei. J Am Soc Mass Spectrom 14: $728-735$.

Panigrahi AK, Ernst NL, Domingo GJ, Fleck M, Salavati R, Stuart KD. 2006. Compositionally and functionally distinct editosomes in Trypanosoma brucei. RNA 12: 1038-1049.

Panigrahi AK, Zikova A, Dalley RA, Acestor N, Ogata Y, Anupama A, Myler PJ, Stuart KD. 2008. Mitochondrial complexes in Trypanosoma brucei: A novel complex and a unique oxidoreductase complex. Mol Cell Proteomics 7: 534-545.

Pelletier M, Read LK. 2003. RBP16 is a multifunctional gene regulatory protein involved in editing and stabilization of specific mitochondrial mRNAs in Trypanosoma brucei. RNA 9: 457-468.

Phadtare S, Inouye M, Severinov K. 2002. The nucleic acid melting activity of Escherichia coli CspE is critical for transcription antitermination and cold acclimation of cells. J Biol Chem 277: 7239-7245.

Rajkowitsch L, Semrad K, Mayer O, Schroeder R. 2005. Assays for the RNA chaperone activity of proteins. Biochem Soc Trans 33: 450456.

Read LK, Myler PJ, Stuart K. 1992. Extensive editing of both processed and preprocessed maxicircle CR6 transcripts in Trypanosoma brucei. J Biol Chem 267: 1123-1128.

Schnaufer A, Panigrahi AK, Panicucci B, Igo RP Jr, Wirtz E, Salavati R, Stuart K. 2001. An RNA ligase essential for RNA editing and survival of the bloodstream form of Trypanosoma brucei. Science 291: 2159-2162. 
Schumacher MA, Karamooz E, Zikova A, Trantirek L, Lukes J. 2006. Crystal structures of T. brucei MRP1/MRP2 guide-RNA binding complex reveal RNA matchmaking mechanism. Cell 126: 701-711.

Shu HH, Stuart K, Goringer HU. 1995. Guide RNA molecules not engaged in RNA editing form ribonucleoprotein complexes free of mRNA. Biochim Biophys Acta 1261: 349-359.

Simpson L, Aphasizhev R, Gao G, Kang X. 2004. Mitochondrial proteins and complexes in Leishmania and Trypanosoma involved in U-insertion/deletion RNA editing. RNA 10: 159-170.

Stuart KD, Schnaufer A, Ernst NL, Panigrahi AK. 2005. Complex management: RNA editing in trypanosomes. Trends Biochem Sci 30: $97-105$.

Sturm NR, Simpson L. 1991. Leishmania tarentolae minicircles of different sequence classes encode single guide RNAs located in the variable region approximately $150 \mathrm{bp}$ from the conserved region. Nucleic Acids Res 19: 6277-6281.
Sturm NR, Maslov DA, Blum B, Simpson L. 1992. Generation of unexpected editing patterns in Leishmania tarentolae mitochondrial mRNAs: Misediting produced by misguiding. Cell 70: 469476.

Vondruskova E, van den Burg J, Zikova A, Ernst NL, Stuart K, Benne R, Lukes J. 2005. RNA interference analyses suggest a transcript-specific regulatory role for mitochondrial RNAbinding proteins MRP1 and MRP2 in RNA editing and other RNA processing in Trypanosoma brucei. J Biol Chem 280: 24292438.

Weng J, Aphasizheva I, Etheridge RD, Huang L, Wang X, Falick AM, Aphasizhev R. 2008. Guide RNA-binding complex from mitochondria of trypanosomatids. Mol Cell 32: 198-209.

Zikova A, Kopecna J, Schumacher MA, Stuart K, Trantirek L, Lukes J. 2008. Structure and function of the native and recombinant mitochondrial MRP1/MRP2 complex from Trypanosoma brucei. Int J Parasitol 38: 901-912. 

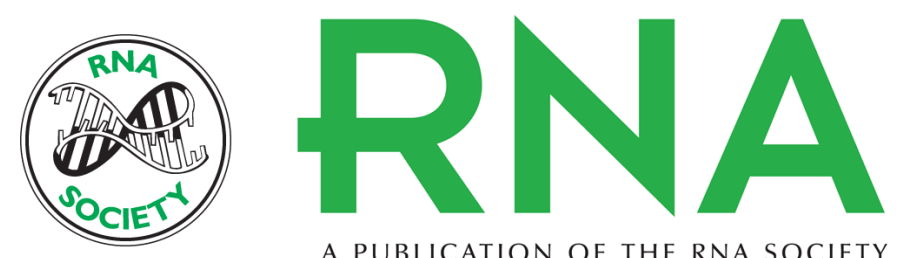

A PUBLICATION OF THE RNA SOCIETY

\section{TbRGG2 facilitates kinetoplastid RNA editing initiation and progression past intrinsic pause sites}

Michelle L. Ammerman, Vladimir Presnyak, John C. Fisk, et al.

RNA 2010 16: 2239-2251 originally published online September 20, 2010

Access the most recent version at doi:10.1261/rna.2285510

\section{Supplemental http://rnajournal.cshlp.org/content/suppl/2010/08/25/rna.2285510.DC1 \\ Material}

References This article cites 46 articles, 25 of which can be accessed free at:

http://rnajournal.cshlp.org/content/16/11/2239.full.html\#ref-list-1

\section{License}

Email Alerting Receive free email alerts when new articles cite this article - sign up in the box at the Service top right corner of the article or click here.

To subscribe to $R N A$ go to:

http://rnajournal.cshlp.org/subscriptions 